This item was submitted to Loughborough's Research Repository by the author.

Items in Figshare are protected by copyright, with all rights reserved, unless otherwise indicated.

\title{
Maintaining control performance in faulty control systems
}

PLEASE CITE THE PUBLISHED VERSION

PUBLISHER

(C) IEEE

VERSION

VoR (Version of Record)

LICENCE

CC BY-NC-ND 4.0

\section{REPOSITORY RECORD}

Dai, Chengwei, and Shuang-Hua Yang. 2019. "Maintaining Control Performance in Faulty Control Systems". figshare. https://hdl.handle.net/2134/4129. 
This item was submitted to Loughborough's Institutional Repository (https://dspace.lboro.ac.uk/) by the author and is made available under the following Creative Commons Licence conditions.

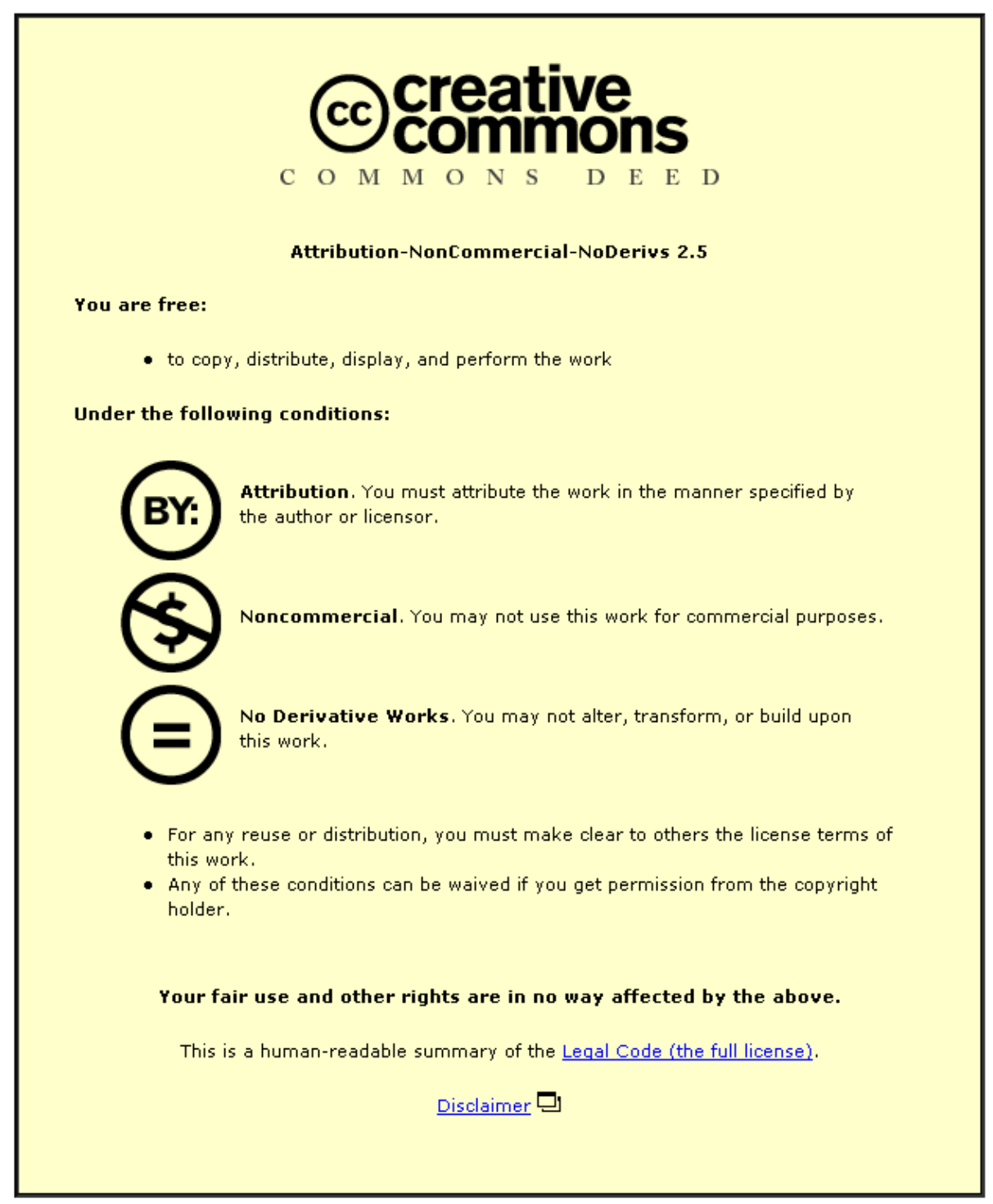

For the full text of this licence, please go to: http://creativecommons.org/licenses/by-nc-nd/2.5/ 


\title{
Maintaining Control Performance in Faulty Control Systems
}

\author{
Chengwei Dai and Shuang $H$. Yang \\ Department of computer science \\ Loughborough Univiersty \\ Loughborough, Leicestershire \\ LE11 3TU UK \\ \{C.Dai, S.H.Yang\}@lboro.ac.uk
}

\begin{abstract}
Controller failures degrade a control system performance. In this paper, a novel maintenance approach for controller failures is proposed to restore the degraded performance of the controller. The method is to equivalently shift any fault occurring in a controller to the plant. Based on the assumed process model, a compensator with a serial link is designed to maintain the faulty controller in the SISO and MIMO control systems. Several simulation results are given to illustrate the procedure of using the method.
\end{abstract}

Keywords: Control performance, fault equivalent transfer, compensator.

\section{Introduction}

Control performance usually refers to how well a controller and the process it controls work together in a single loop. This topic is of importance to control specialists and process engineers who have inherited the control systems within their plants. In actual applications, a controller is expensive to implement and maintain; many factors can contribute to their abrupt or gradual performance deterioration, including sensor/actuator failures, equipment fouling, controller failure, and product change.

Previous works addressing on the maintenance of control performance often focuses on the hardware failure in control systems. In the last decade, Fault Tolerant Controls (FTCs) have enjoyed tremendous successful applications to effectively accommodate defects in sensors, actuators or plants to maintain the performance in an acceptable range. A number of theoretical results as well as application examples have been described in the literature $[1,2,3]$.

However, few of them considered what should be done to the degraded performance due to controller failures. The wide implementation of advanced control strategies in the control systems to handle various difficult situations makes controllers more and more complex, which directly leads to the increase of proneness to failure. Due to the crucial role of controllers in control systems, minor failures can cause disastrous damage; therefore, the failure must be mitigated and control systems must be maintained when a fault occurs.

The aim of this paper is to maintain a faulty controller and restore its degraded control performance. A novel fault transfer approach is proposed, which shifts faults occurring in the controller to the plant; a compensator with a serial link is designed to maintain the assumed faulty plant and then transferred into the controller according to the equality of the control loop transfer function. The proposed method is applicable to SISO and MIMO control systems. Several simulation results are given to illustrate the procedure of using the method.

The rest of this paper is arranged as follows. A description of fault equivalent transfer is presented in Section 2 followed by the compensator design in Section 3. Section 4 extends the proposed maintenance approach to MIMO control systems. Section 5 presents several simulation results to illustrate the proposed maintenance approach. Section 6 gives the conclusions.

\section{Fault Equivalent Transfer}

Traditional FTCs handling plant failures heavily rely on a post-fault process model. On basis of the post-fault process model, a reconfigurable control is designed to update the existing controller and compensate for the effect of the fault. Different from plants or machines, whose models are estimated by system identification techniques, advanced controllers such as Model Predictive Controls (MPCs) are working in a wide operation range and their parameters are real time updated in response to controlled process dynamics to achieve a time-varying objective function; therefore, it is hard to establish an accurate mathematical model as a post-fault model to precisely describe controller faulty behaviors.

\footnotetext{
• 0-7803-8566-7/04/\$20.00 @ 2004 IEEE.
} 
In this section, we introduce a novel approach to estimate the post-fault model. Consider a feedback control system shown in Fig.1, in which $G_{c}$ represents a controller model, $G_{p}$ is a plant model. Assume that a fault occurs in the controller and the fault model can be described as $G_{f}$; set $m$ as the identified model for the normal open loop between the controller input, $e(t)$, and the process output $y(t)$ and $m_{f}$ as the identified model for the faulty open loop.

$$
\begin{gathered}
\left\{\begin{array}{l}
m(s) \approx G_{p}(s) G_{c}(s) \\
m_{f}(s) \approx G_{p}(s) G_{f}(s) G_{c}(s)
\end{array}\right. \\
G_{f}(s) \text { is calculated by } \\
G_{f}(s)=\frac{m_{f}(s)}{m(s)}
\end{gathered}
$$

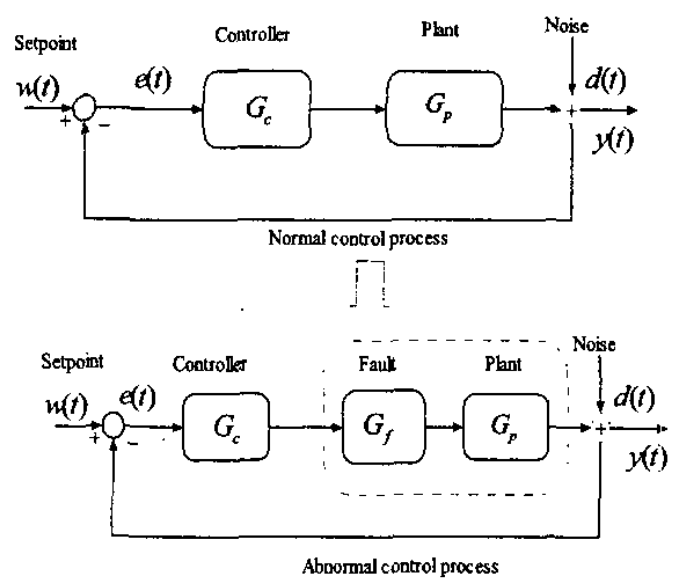

Figure 1. Normal and abnormal control loops

Working on the transfer function of the open control loop, there is no difference whether a fault occurred in the controller or in the plant, and the model of the open loop remains $G_{p}(s) G_{f}(s) G_{c}(s)$. Therefore, when a controller failure happens, we can suppose the controller is still in a normal state, the fault part $G_{f}$ is "shifted" to the plant and the equivalent faulty plant model is:

$$
G_{\text {new }}(s)=G_{p}(s) G_{f}(s)
$$

This idea also can extend to other components in the control systems; faults in these components can also be transferred to the plant with the model $G_{\text {new }}$.

\section{Compensator Design}

To maintain the degraded control performance in the case of a controller failure, a serial link compensator is designed here. The objective of the compensator is to work together with the faulty controller to stabilize the control process and restore the degraded performance.

The design of the compensator consists of two steps: 1. Designing a new controller. Based on the post-fault process model $G_{n e w}$, we can design a new controller $\widetilde{G}_{c}$ for the equivalent faulty plant to achieve a satisfactory control performance.

2. Calculating the compensator model. Assume the compensator $G_{\text {com }}$ is in a serial link with the existing controller, as shown in Fig. 2, the designed compensator works with the existing faulty controller to take the place of the new controller $\widetilde{G}_{c}$ in order to maintain the degraded performance.

The model of the compensator is given as follows:

$$
\left\{\begin{array}{l}
{\left[1+G_{c o m}(s)\right] \times G_{c}(s)=\tilde{G}_{c}(s)} \\
G_{c o m}(s)=\frac{\tilde{G}_{c}(s)-G_{c}(s)}{G_{c}(s)}
\end{array}\right.
$$

and the transfer function of the open loop is $G_{p}(s) G_{f}(s) G_{c}(s)\left[1+G_{c o m}(s)\right]$.

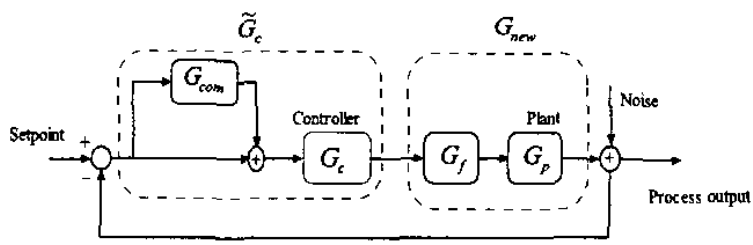

(a). Compensator with the fault in the plant

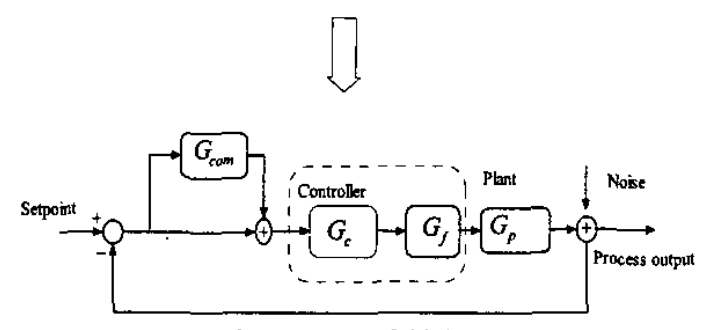

(b). Compensator with the fauk in the controlit

Figure 2. Serial link compensator

It is obvious that the original controller $G_{c}(s)$ and the faulty part of the controller $\mathrm{G}_{\mathrm{f}}(\mathrm{s})$ can be reunified as Fig.2 (b). It means that the compensator designed for the equivalent faulty plant $G_{\text {new }}(\mathrm{s}$ ) in Fig.2 (a) can be adopted directly for the faulty controller to maintain its performance. 


\section{Extension to MIMO Systems}

Consider a faulty control system shown in Fig.3 (a) and set $\mathbf{M}_{f}$ as the estimated model for the abnormal open loop. In the case of a controller failure, we treat the faulty controller being in a normal state, but transfer the controller faults into the plant. Suppose there is a $\operatorname{model} \widetilde{\mathbf{G}}_{f}$ so that:

$$
\widetilde{\mathbf{G}}_{f} \mathbf{G}_{p}=\mathbf{G}_{p} \mathbf{G}_{f}
$$

Therefore, the faulty control loop can be reconstructed as shown in Fig.3 and $\mathbf{M}_{f}$ can be rewritten as: $\mathbf{M}_{f} \approx \widetilde{\mathbf{G}}_{f} \mathbf{G}_{p} \mathbf{G}_{c}$

The fault model $\mathbf{G}_{f}$ in fact doesn't exist in the actual control system; it is only an alternative way to mathmatically describe the fault. Therefore transferring the model $\mathbf{G}_{f}$ into $\widetilde{\mathbf{G}}_{f}$ is reasonable.

$\widetilde{\mathbf{G}}_{f}$ can be obtained as follows: assume there are two group data available, one for the normal state of the open loop, the other for the fault state. $\mathbf{E}_{n}$ and $\mathbf{Y}_{n}$ are the nominal inputs and outputs of the open loop, $\mathbf{E}_{f}$ and $\mathbf{Y}_{f}$ are the faulty inputs and outputs. Set $\mathbf{M}_{1}^{-1}$ as the estimated inverse model for the normal open loop, which can be identified by this data group: $\left\{\mathbf{E}_{n}, \mathbf{Y}_{n}\right\} \rightarrow \mathbf{M}_{1}^{-1}$

$\mathbf{M}_{1}^{-1} \approx\left[\mathbf{G}_{p} \mathbf{G}_{c}\right]^{-1}$

$\mathbf{M}_{f}$ can be estimated by identifying the group of data :

$$
\left\{\mathbf{Y}_{f}, \mathbf{E}_{f}\right\} \rightarrow \mathbf{M}_{f}
$$

Therefore we have

$$
\begin{aligned}
& \widetilde{\mathbf{G}}_{f}=\mathbf{M}_{f} \mathbf{M}_{1}^{-1} \\
& \mathbf{G}_{\text {new }}=\widetilde{\mathbf{G}}_{f} \mathbf{G}_{p}
\end{aligned}
$$

Based on the equivalent faulty process model, $\mathbf{G}_{n e w}$, we can design a new controller $\widetilde{\mathbf{G}}_{c}$ to govern the equivalent plant. Therefore, the compensator model in MIMO can be obtained according to Equation 4 :

$\mathbf{G}_{c o m}=\frac{\widetilde{\mathbf{G}}_{c}}{\mathbf{G}_{c}}-\mathbf{I}=\mathbf{G}_{p} \widetilde{\mathbf{G}}_{c} \mathbf{M}_{1}^{-1}-\mathbf{I}$

where $\mathbf{I}$ is a unit matrix.

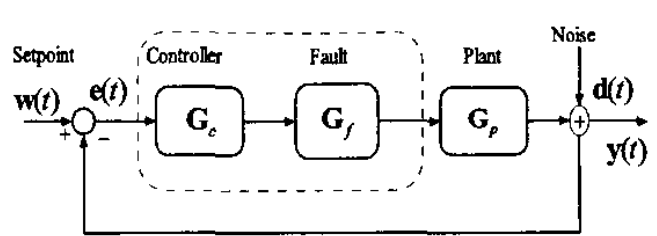

(a) Faulty Controber

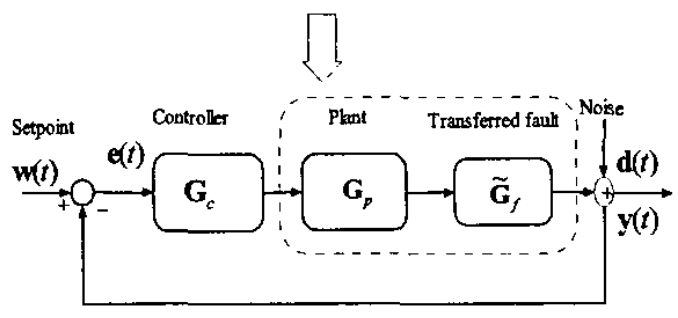

(b). Fault Transfer

Figure 3. Transferring controller fault

\section{Simulation Results}

\subsection{SISO case study}

The simulation of a water tank process is implemented by using MATLAB. The transfer function of the water tank is $G_{p}=\frac{0.1}{\mathrm{z}-0.8}$. The process is governed by a discrete PID controller with the parameters $\mathrm{K}_{\mathrm{p}}=8$, $\mathrm{K}_{\mathrm{I}}=1, \mathrm{~K}_{\mathrm{D}}=0.001$. The sampling interval is 1 second. The transfer function of the PID controller is: $G_{c}=\frac{17 z^{2}+1.992 z-15}{2 z^{2}-2}$.

A fault occurred at the instant 400 in which a constant gain is attached in the PID output outlet, $k=0.1$, and made the output of the PID controller becoming $u_{P D D_{-} f}=u_{P I D} \times 0.1$, where $u_{P I D}$ is the control signals of the PID controller in the normal state, $u_{P I D_{-} f}$ is the controller signal in the faulty case.

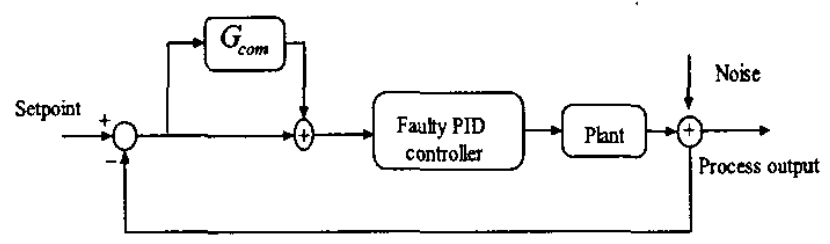

Figure 4. Serial link compensator for a PID controller 


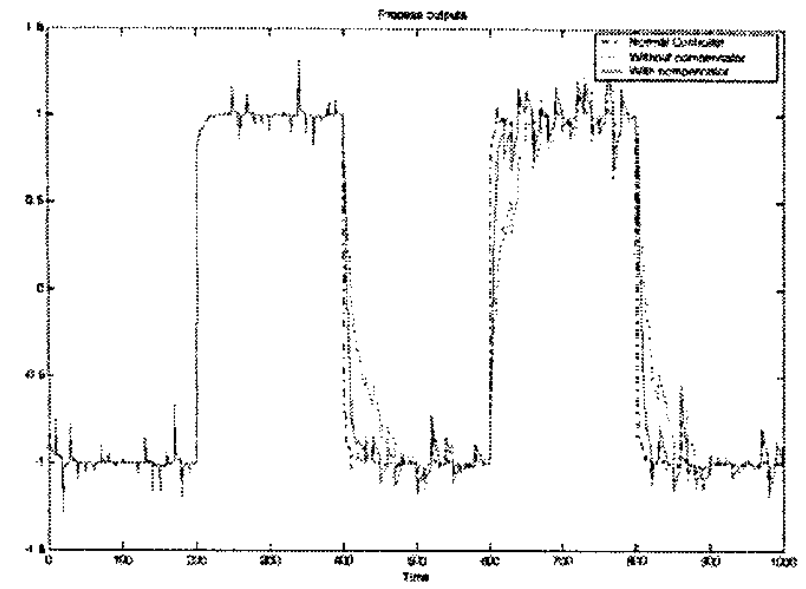

Figure 5. Faulty PID controller with and without compensation

To be simple, in this simulation, we directly use the faulty model $G_{f}=0.1$ and transfer the fault to the plant; the equivalent faulty process model $G_{\text {new }}$ is obtained: $G_{n e w}=\frac{0.01 z}{z-0.8}$.

According to $G_{n e w}$, we can design a new PI controller to govern the process with the parameters: $\mathrm{K}_{\mathrm{p}}=10, \mathrm{~K}_{\mathrm{l}}=0.1$ and the sampling rate is 1 second. The transfer function of the PI controller is: $\widetilde{G}_{c}=\frac{20.1 z-19.9}{2 z-2}$.

Based on Equation 4, the model of the compensator is calculated as:

$$
G_{\text {com }}=\frac{-6.192 \mathrm{z}^{3}+9.776 \mathrm{z}^{2}+6.224 \mathrm{z}-9.808}{40.2 \mathrm{z}^{3}-39.8 \mathrm{z}^{2}-40.2 \mathrm{z}+39.8}
$$

The serial link compensator is implemented to maintain the faulty PID controller as shown in Fig 4. Fig. 5 indicates that the degraded performances are recovered after the compensator is added in the process at the instant 400 ; the outputs with the compensator are able to track the setpoint satisfactorily.

\subsection{MIMO case study}

Consider a 2-input-2-ouptut control process, the plant model is:

$$
\mathbf{G}_{p}=\left[\begin{array}{cc}
\frac{1}{s+3} & \frac{1}{s^{2}+4 s+3} \\
\frac{2}{s^{2}+4 s+3} & \frac{1}{s^{2}+4 s+3}
\end{array}\right]
$$

A feedback controller is designed to govern the process; the controller model is:
$\mathbf{G}_{c}=\left[\begin{array}{cc}\frac{4 s^{3}+20 s^{2}+32 s+16}{2 s^{3}+11 s^{2}+23 s+20} & \frac{4 s^{2}+24 s+32}{2 s^{3}+17 s^{2}+62 s+80} \\ \frac{-4 s^{3}-16 s^{2}-20 s-8}{2 s^{3}+11 s^{2}+23 s+20} & \frac{8 s^{3}+56 s^{2}+112 s+64}{2 s^{3}+17 s^{2}+62 s+80}\end{array}\right]$

At the instant 315 , a fault occurred in the controller, which is a constant gain matrix:

$\mathbf{G}_{f}=\left[\begin{array}{cc}0.1 & 0 \\ 0 & 0.1\end{array}\right]$

It is attached on the controller output and leads to the process output deviating away from the desired range.

To maintain the faulty controller, we identify the normal open loop model of the process:

$\mathbf{M}_{1}^{-1}=\left[\begin{array}{cc}\frac{0.7268}{s+0.2167} & 0 \\ \frac{0.141}{s+0.2167} & 0\end{array}\right]$

The faulty open loop model matrix is estimated as:

$\mathbf{M}_{f}=\left[\begin{array}{cc}\frac{0.5036}{4.919 \mathrm{~s}+15.92} & 0 \\ \frac{0.763}{4.919 \mathrm{~s}+15.92} & 0\end{array}\right]$

$\widetilde{\mathbf{G}}_{f}$ can be calculated according to Equation 8:

$\tilde{\mathbf{G}}_{f}=\left[\begin{array}{cc}\frac{0.366}{4.919 \mathrm{~s}^{2}+16.99 \mathrm{~s}+3.45} & 0 \\ \frac{0.55}{4.919 \mathrm{~s}^{2}+16.99 \mathrm{~s}+3.45} & 0\end{array}\right]$

The step responses of $\widetilde{\mathbf{G}}_{f} \mathbf{G}_{p}$ and $\mathbf{G}_{p} \mathbf{G}_{f}$ are shown in Fig 6, which illustrates that the two models are approximately identical .

Applying $\widetilde{\mathbf{G}}_{f}$ into Equation 9 , the equivalent fault process model $\mathbf{G}_{\text {new }}$ is obtained:

$\mathbf{G}_{m \mathrm{~m}}=\left[\begin{array}{cc}\frac{0.5655}{4.9 \mathrm{~s}^{3}+31.7 \mathrm{~s}^{2}+54.4 \mathrm{~s}+10.3} & \frac{0.5655}{4.9 \mathrm{~s}^{4}+31.7 \mathrm{~s}^{3}+74.1 \mathrm{~s}^{2}+78.3 \mathrm{~s}+13.8} \\ \frac{1.052}{4.9 \mathrm{~s}^{3}+31.7 \mathrm{~s}^{2}+54.4 \mathrm{~s}+10.3} & \frac{1.052}{4.9 \mathrm{~s}^{4}+31.7 \mathrm{~s}^{3}+74.1 \mathrm{~s}^{2}+78.3 \mathrm{~s}+13.8}\end{array}\right]$

Based on $\mathbf{G}_{n e w}$, a new LQG controller $\widetilde{\mathbf{G}}_{c}$ is designed, and the controller model is given:

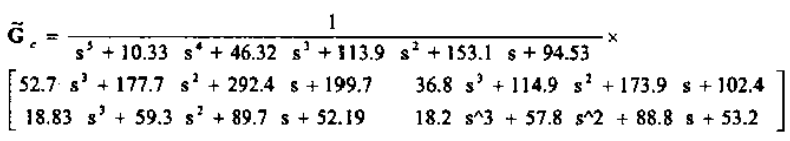



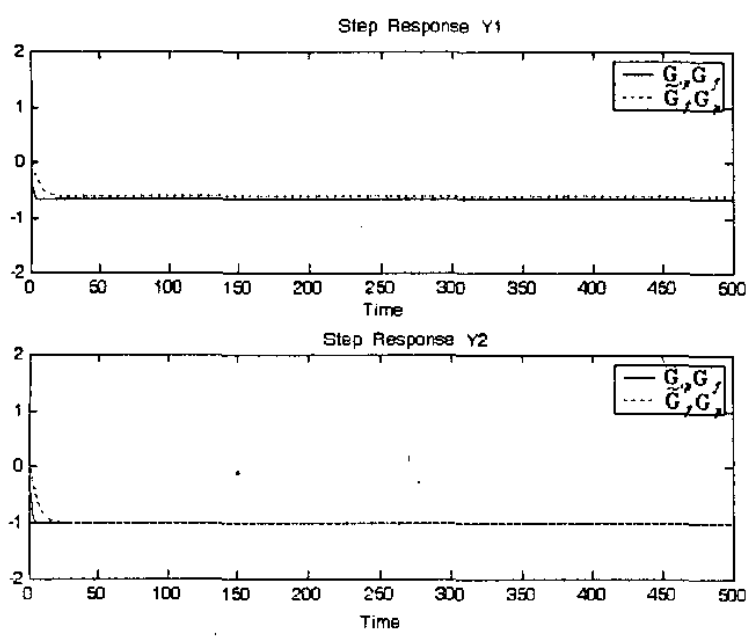

Figure 6. Step response
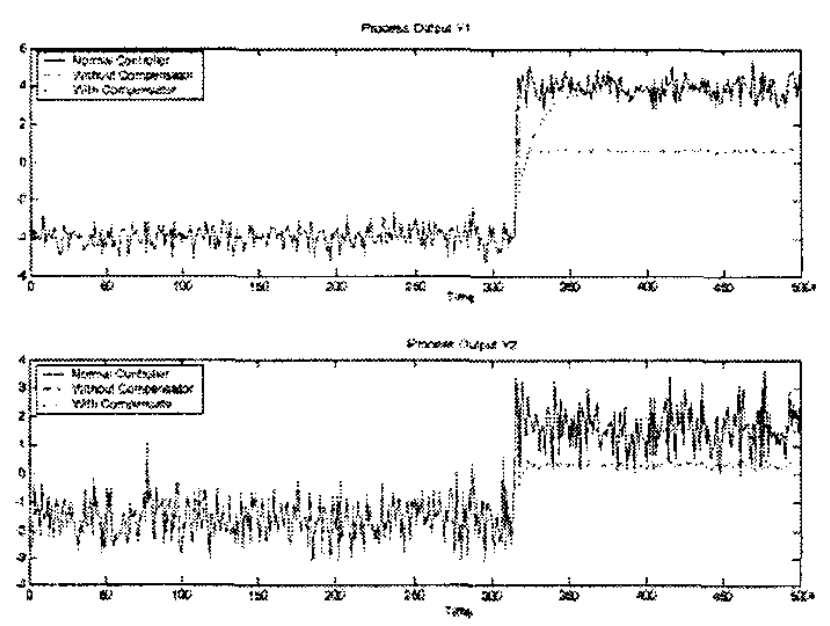

Figure 7. Faulty control process with and without compensation

According to Equation 10, the model of the compensator is calculated and simplified as:

$\mathbf{G}_{\text {com }}=\left[\begin{array}{ll}\frac{0.2283 \mathrm{~s}+0.5045}{\mathrm{~s}^{2}+0.5995 \mathrm{~s}+0.0616} & 0 \\ \frac{-0.035 \mathrm{~s}+0.06334}{\mathrm{~s}^{2}+0.5995 \mathrm{~s}+0.0616} & 0\end{array}\right]$

Implementing the compensator to the faulty control process, the results in Fig. 7 show that the degraded performance is satisfactorily recovered.

\section{Conclusions and Future Works}

In this paper, we have presented a novel approach to maintain the degraded control performance in the case of controller failures. This approach is based on the fault transfer, which shifts any fault occurring in the controller to the plant. A compensator is designed to maintain the control performance for the equivalent faulty plant and then transferred into the controller failure scenario. The proposed method is applicable to SISO and MIMO control systems. The distinguished feature of the maintenance method is that it extends the range of traditional FTCs and focuses on the controller. This method is not only able to maintain the controller failures but also can extend to attack the poor controller parameter setting in order to maintain the optimum performance of the control systems.

Recent progress in the Internet technology inspires the prospect for the remote maintenance. We are implementing the proposed method for remotely maintenance of local controllers with the support of the Internet technologies.

\section{References}

[1] Y. Zhang, and J. Jiang, "Fault Tolerant Control System Design with Explicit Consideration of Performance Degradation," IEEE Transactions on aerospace and electronic system, vol. 39, no. 3, pp. 838-848, July 2001.

[2] H. Niemann H. and J. Stoustrup "Controller Reconfiguration based on LTR Design" 42nd IEEE Conference on Decision and Control 2003, Hawaii, USA.

[3] Y. Zhang, and J. Jiang, "Integrated design of reconfigurable fault tolerant control systems". Journal of Guidance, Control, and Dynamics, 24, 1, (Jan.-Feb. 2001), 133-136.

[4] E. C. Kerrigan and J. M. Maciejowski, "FaultTolerant Control of a Ship Propulsion System using Model Predictive Control", Proceedings of the European Control Conference, Karlsuhe, Germany, August 1999.

[5] Jan M. Maciejowski and Colin N. Jones, "MPC FaultTolerant Flight Control Case Study: Flight 1862", IFAC Safe Process Conference, Washington DC, 9-11 June 2003.

[6] N. EvaWu, "Coverage in Fault TolerantControl", http://www.ws.binghamton.edu/wuweb/papers/WuAutoma tica04.pdf

[7] C. De Persis and A. Isidori, "A geometric approach to nonlinear fault detection and isolation", IEEE Transaction on Automatic Control, AC-46, pp. 853-865, 2001. 